\title{
Aprendizagem de Relações Ordinais por meio de Treino de uma única Sequência de Estímulos
}

\author{
Grauben José Alves de Assis ${ }^{1}$ \\ Universidade Federal do Pará \\ Diogo Rodrigues Corrêa \\ Universidade Federal do Pará \\ Carlos Barbosa Alves de Souza \\ Universidade Federal do Pará \\ Paulo Sérgio Teixeira Prado \\ Universidade Estadual Paulista - Campus de Marília
}

\begin{abstract}
RESUMO - O objetivo do estudo foi avaliar a formação de classes ordinais a partir do ensino de uma única sequência de estímulos sob controle da numerosidade. Participaram cinco crianças na faixa etária de 4 a 5 anos. Os estímulos foram formas abstratas referentes à numerosidade de 1 a 5 . Usou-se um procedimento de ensino por sobreposição de estímulos. Todos os participantes alcançaram o critério de acerto na linha de base. Nos testes de transitividade e conectividade, os cincos participantes responderam prontamente. Houve generalização para duas novas classes ordinais. Nos testes de manutenção três participantes apresentaram responder consistente e um dos participantes respondeu parcialmente. Este estudo é uma contribuição para o estabelecimento de uma análise funcional da aprendizagem de repertórios numéricos.
\end{abstract}

Palavras-chave: relações ordinais; sobreposição de estímulos; crianças.

\section{Learning of Ordinal Relations through the Training of one Sequence of Stimuli}

\begin{abstract}
The aim of this study was to evaluate the learning of ordinal classes through the training of only one sequence of stimuli. Five children, four to five years old participated. Sets of stimuli with abstract shapes, representing the numerical sequence from one to five were presented via a microcomputer. Once the criteria for success were reached, tests were administered. All of the participants reached the criteria for success during the baseline phase, and responded readily to the tests for transitivity and connectivity. Generalization also occurred for two novel ordinal classes. During the retest phase, three children responded consistent with prior training, whereas one child responded partially. This study is a contribution to a functional analysis of the process of learning of numeric repertoires.
\end{abstract}

Keywords: ordinal relations; stimulus overlapping; children.

Responder sequencial ou ordinalmente na presença de estímulos apresentados simultaneamente implica responder sob o controle da propriedade relacional 'ordem dos estímulos'. A aprendizagem desse tipo de comportamento se mostra relevante no contexto da aprendizagem humana devido à sua relação com a aquisição de habilidades matemáticas (Carmo, 2002; Kahhale, 1993; Prado \& de Rose, 1999) e lingüísticas (Ribeiro, Assis \& Emuno, 2005). Por exemplo, uma criança que está aprendendo a falar terá suas vocalizações reforçadas diferencialmente e, portanto, modeladas, em função do ajuste das mesmas à sequência de sons definidos por sua comunidade verbal. Quando ela começar a aprender a contar deverá responder à sequência dos números.

De acordo com Green, Stromer e Mackay (1993) um aspecto interessante da aprendizagem do repertório de responder ordinalmente é que uma vez estabelecido para os componentes de um determinado estímulo ou de um conjunto de estímulos (por meio de contingências de reforçamento simples, i.e. de três termos), ele possibilita a emergência de

1 Endereço para correspondência: Rua Jerônimo Pimentel, 426/1801, Umarizal. Belém, PA. CEP: 66055-000. Fone: (91) 3201-7662. E-mail: ggrauben@gmail.com novas respostas ordinais para os elementos do estímulo ou do conjunto sem a necessidade de treinos adicionais. Segundo estes autores isto ocorre porque as relações ordinais estabelecidas pelas contingências de reforçamento simples apresentam quatro propriedades, definidas a partir das propriedades das relações de ordinalidade na matemática. A primeira é a Irreflexividade, que determina que em uma sequência um estímulo não pode ser seguido por ele mesmo, em função de sua posição ordinal: por exemplo, estabelecida a relação $\mathrm{A} 1 \rightarrow \mathrm{A} 2$ (A1 é seguido por $\mathrm{A} 2$ ) as relações ordinais $\mathrm{A} 1 \rightarrow \mathrm{A} 1$ e $\mathrm{A} 2 \rightarrow \mathrm{A} 2$ não são possíveis. A segunda propriedade é a Assimetria, que estabelece a unidirecionalidade dos estímulos em uma relação ordinal: por exemplo, estabelecida a relação $\mathrm{A} 1 \rightarrow \mathrm{A} 2$, sua reversão $(\mathrm{A} 2 \rightarrow \mathrm{A} 1)$ não é possível nesta relação. A terceira propriedade é a Transitividade, que determina que uma vez estabelecidas relações ordinais entre pelo menos dois pares de estímulos não adjacentes, havendo um estímulo em comum nos pares, se estabelece também uma relação ordinal entre os outros estímulos: por exemplo, ensinadas as relações $\mathrm{A} 2 \rightarrow \mathrm{A} 3$ e $\mathrm{A} 3 \rightarrow \mathrm{A} 4$, fica estabelecida a relação ordinal $\mathrm{A} 2 \rightarrow \mathrm{A} 4$. A quarta propriedade é a Conectividade, que estabelece que todos os pares de estímulos de uma 
relação ordinal aprendida adquirem relações ordinais entre si: por exemplo, ensinada a relação ordinal $\mathrm{A} 1 \rightarrow \mathrm{A} 2 \rightarrow \mathrm{A} 3$, ficam estabelecidas as relações $\mathrm{A} 1 \rightarrow \mathrm{A} 2, \mathrm{~A} 1 \rightarrow \mathrm{A} 3$ e A2 $\rightarrow \mathrm{A} 3$.

Aplicando a proposta de Green e cols. (1993), Stromer e Mackay (1993) desenvolveram um estudo com duas crianças e cinco adultos, procurando avaliar a emergência de novas sequências a partir do ensino de duas ou mais sequências de estímulos apresentados na tela de um computador. Foram realizados dois experimentos com treino por sobreposição de estímulos utilizando o procedimento de fading in para indicar qual estímulo deveria ser tocado primeiro na tela do computador. No treino por sobreposição os estímulos eram apresentados aos pares e à medida que um novo estímulo era adicionado na formação de uma nova sequência o primeiro elemento da sequência anterior era retirado. Dessa maneira, os elementos que formavam a sequência completa nunca apareciam juntos durante o treino. Por exemplo, para se treinar a sequência $\mathrm{A} 1 \rightarrow \mathrm{A} 2 \rightarrow \mathrm{A} 3 \rightarrow \mathrm{A} 4 \rightarrow \mathrm{A} 5$, se iniciava pelo treino de $\mathrm{A} 1 \rightarrow \mathrm{A} 2$, em seguida $\mathrm{A} 2 \rightarrow \mathrm{A} 3$, e assim por diante.

No primeiro experimento Stromer e Mackay (1993) verificaram se participantes que foram ensinados a responder em sequência a um conjunto de cinco estímulos $(\mathrm{A} 1 \rightarrow \mathrm{A} 2 \rightarrow \mathrm{A} 3 \rightarrow \mathrm{A} 4 \rightarrow \mathrm{A} 5)$ respondiam sequencialmente aos pares não adjacentes daquele conjunto, de forma consistente com o treino $($ e.g. $\mathrm{A} 1 \rightarrow \mathrm{A} 3, \mathrm{~A} 1 \rightarrow \mathrm{A} 4, \mathrm{~A} 2 \rightarrow \mathrm{A} 4$, etc). A produção de sequências de dois estímulos sugeriria que esse desempenho estava baseado no estabelecimento de uma relação ordinal entre os cinco estímulos. $\mathrm{O}$ experimento também buscou verificar se treinar dois conjuntos (A e B) de cinco estímulos permitiria a produção de novas sequências contendo os estímulos de ambos os conjuntos. Este último ponto buscava verificar a substituibilidade dos estímulos, ou seja, se o participante responderia aos estímulos em função da sua ordem na relação ordinal estabelecida, independentemente de a qual conjunto ele pertencia. Um segundo experimento procurou ensinar outra sequência de cinco estímulos $(\mathrm{C} 1 \rightarrow \mathrm{C} 2 \rightarrow \mathrm{C} 3 \rightarrow \mathrm{C} 4 \rightarrow \mathrm{C} 5)$ e testar a sua inclusão nas duas outras sequências estabelecidas no primeiro experimento.

O estudo mostrou o desenvolvimento de novas sequências de cinco estímulos e a expansão de classes de sequência após ensino com sobreposição. Segundo os autores, a variação do procedimento a partir do ensino de pares adjacentes de estímulos pode fornecer uma maneira de isolar e avaliar propriedades transitivas potenciais de relações ordinais entre estímulos.

Stromer, Mackay, Cohen e Stoddard (1993) replicaram o estudo de Stromer e Mackay (1993) com dois participantes com retardo mental e uma criança de cinco anos da pré-escola com desenvolvimento típico. O desempenho dos participantes foi consistente com o treino e sugeriu a formação de classes de sequência de estímulos mutuamente substituíveis. Esse estudo foi relevante por estender os resultados anteriores para indivíduos com limitações no desenvolvimento cognitivo.

Holcomb, Stromer e Mackay (1997) utilizando o mesmo procedimento de Stromer e cols. (1993) - sobreposição de estímulos - buscaram verificar a formação de novas sequências não diretamente ensinadas. Quinze crianças participaram do estudo dividido em três experimentos. No Experimento 1, cada criança foi preliminarmente ensinada a responder a uma sequência de seis estímulos. Em seguida, com outro conjunto de estímulos, treinou-se a ordenação de pares de estímulos e testou-se a produção de novas sequências com pares de estímulos não adjacentes. No Experimento 2, acrescentaram-se consequências diferenciais após cada tentativa de teste. No Experimento 3, investigou-se o comportamento verbal das crianças, para as quais foram feitas perguntas para avaliar os critérios que elas usaram na escolha dos estímulos. O estudo mostrou que o procedimento por sobreposição de estímulos pode produzir novas sequências com três ou mais estímulos.

Galy, Camps e Melan (2003) examinaram com estudantes universitários a substituibilidade dos estímulos, a partir do ensino de três sequências curtas $(\mathrm{A} 1 \rightarrow \mathrm{A} 2 \rightarrow \mathrm{A} 3, \mathrm{~B} 1 \rightarrow \mathrm{B} 2 \rightarrow \mathrm{B} 3$ e $\mathrm{C} 1 \rightarrow \mathrm{C} 2 \rightarrow \mathrm{C} 3$ ) por encadeamento de respostas, cujos estímulos foram introduzidos gradativamente (primeiro A1, então $\mathrm{A} 1 \rightarrow \mathrm{A} 2$, em seguida $\mathrm{A} 1 \rightarrow \mathrm{A} 2 \rightarrow \mathrm{A} 3$ ). Três sequências diferentes foram ensinadas a partir de três conjuntos de estímulos cada $(\mathrm{A}=$ cores; $\mathrm{B}=$ barras paralelas e $\mathrm{C}=$ formas geométricas). Os autores usaram um quarto estímulo (com a função de distrator) na linha de base $(\mathrm{A} 1 \rightarrow \mathrm{A} 2 \rightarrow \mathrm{A} 3 / \mathrm{A} 4$; $\mathrm{B} 1 \rightarrow \mathrm{B} 2 \rightarrow \mathrm{B} 3 / \mathrm{B} 4$; e $\mathrm{C} 1 \rightarrow \mathrm{C} 2 \rightarrow \mathrm{C} 3 / \mathrm{C} 4)$. Os testes de substituição múltipla envolveram um estímulo de cada sequência de linha de base e um estímulo distrator (e.g. A1 $\rightarrow \mathrm{C} 2 \rightarrow \mathrm{B} 3 / \mathrm{B} 4)$. Segundo os autores, esse tipo de arranjo experimental nos testes evitou que os participantes agrupassem os estímulos pelos seus conjuntos correspondentes como ocorreu no estudo de Stromer e Mackay $(1993)($ e.g. A1 $\rightarrow$ A3 $\rightarrow$ B2 $\rightarrow$ B4 $\rightarrow$ B5) que não evidenciou a substituibilidade de estímulos.

Os resultados dos testes de substituibilidade de estímulos evidenciaram um desempenho consistente com a formação de classes ordinais. Ou seja, um responder sob controle da posição (primeira, segunda, terceira) que cada estímulo exerceu na sequência e, como afirmam os autores "na ausência de qualquer feedback consecutivo à resposta (p. 644)".

Os participantes apresentaram um desempenho consistente com o desenvolvimento de classes de sequências. Esse experimento além de oferecer uma alternativa de método de ensino, utilizando o procedimento de encadeamento, levantou questões sobre os efeitos de instruções e feedback consecutivo à resposta a serem testadas em estudos posteriores.

Souza, Assis e Magalhães (2005) demonstraram a emergência de classes ordinais a partir do ensino por sobreposição de estímulos envolvendo três sequências diferentes (formas abstratas nas quantidades 1 a 5, numerais em Libras correspondentes a estes valores e nomes dos numerais). No Experimento 1, cinco crianças surdas foram submetidas a testes de transitividade e conectividade após ordenar cada uma das sequências de estímulos de um a cinco com reforçamento social. Os resultados dos testes mostraram consistência com as contingências de treino. No Experimento 2, três outras crianças surdas responderam consistentemente com as contingências de treino em testes de generalização e re-teste após um período sem contato com as contingências de treino e teste.

Os resultados de Souza e cols. (2005) corroboram os achados de estudos anteriores, os quais apontam na direção de a equivalência pode ser atestada mesmo em contextos que não o de contingência de quatro termos, como no caso do procedimento de emparelhamento com modelo (Green 
\& cols., 1993; Ribeiro \& cols., 2005; Sidman, 1994). Além disso, esses resultados sugerem que a manutenção e a generalização do comportamento ordinal emergente podem ser favorecidas pelo treino de pelo menos uma sequência de estímulos que sobrepõem à contingência de ordem de respostas a propriedade de numerosidade dos estímulos.

Nos estudos mencionados anteriormente o comportamento ordinal emergente foi observado após o treino de pelo menos duas sequências de estímulos. Uma possibilidade ainda não investigada é se o mesmo fenômeno ocorreria a partir do treino de uma única sequência; procedimento que poderia simplificar o treino deste tipo de repertório.

O presente estudo procurou verificar o efeito de um procedimento de ensino com apenas uma sequência de estímulos sobre a aprendizagem de relações ordinais. Além disso, buscou-se verificar a manutenção desse desempenho após exposição do participante às contingências de treino e testes.

\section{Método}

\section{Participantes}

Participaram do estudo cinco pré-escolares com desenvolvimento típico (ver Tabela 1), recrutados por meio de contatos pessoais com os responsáveis, os quais assinaram um termo de consentimento livre e esclarecido autorizando sua participação no estudo. Os participantes freqüentavam uma creche e moravam num conjunto habitacional de baixa-renda.

Tabela 1. Relação dos participantes por gênero e idade.

\begin{tabular}{ccc}
\hline Participantes & Gênero & Idade* $^{*}$ \\
\hline HEN & $\mathrm{F}$ & $5 \mathrm{a} 6 \mathrm{~m}$ \\
WEL & $\mathrm{M}$ & $5 \mathrm{a} 4 \mathrm{~m}$ \\
WAL & $\mathrm{M}$ & $4 \mathrm{a} 3 \mathrm{~m}$ \\
LIE & $\mathrm{M}$ & $4 \mathrm{a} 8 \mathrm{~m}$ \\
DUD & $\mathrm{F}$ & $5 \mathrm{a} 0 \mathrm{~m}$ \\
\hline
\end{tabular}

$* \mathrm{a}=$ anos; $\mathrm{m}=$ meses

\section{Ambiente experimental e equipamento}

As sessões experimentais foram conduzidas em uma sala do laboratório de psicologia da Universidade Federal do Pará, medindo aproximadamente $6 \mathrm{~m}^{2}$, iluminada artificialmente, climatizada e com relativo isolamento acústico. Um microcomputador Pentium IV de $300 \mathrm{MHz}$, com monitor de vídeo dotado de tela sensível ao toque foi usado para "rodar" um programa (REL 4.0 - Santos, Silva, Baptista, \& Assis, 1997) elaborado em Visual Basic 6.0, o qual controlou a apresentação dos estímulos, o intervalo entre tentativas, registrou e conseqüenciou as respostas dos participantes.

\section{Estímulos}

Formas não representacionais na cor preta sobre fundo branco foram utilizadas como estímulos. Três classes (A, B e C) foram definidas de acordo com cada uma de três diferentes formas, tal como ilustração na Figura 1. Os estímulos foram apresentados em números variando de um a cinco. Adotaremos uma notação alfanumérica, sendo que as letras representarão a classe e os numerais o número de estímulos. Por exemplo, B4 indica quatro estímulos da classe B, A2 dois estímulos da classe A e assim por diante. Além disso, houve também variação nas propriedades tamanho - nos valores grande e pequeno - e disposição espacial, que podia ser alinhada e não alinhada.
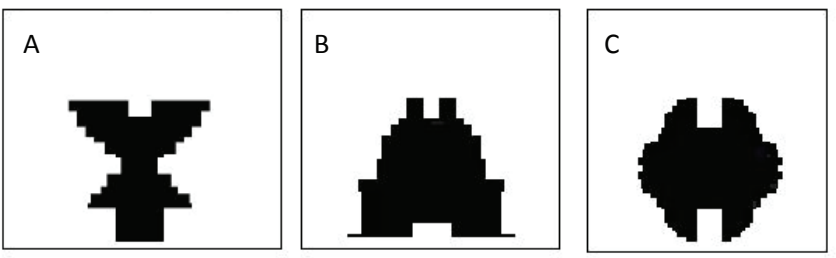

Figura 1. Exemplos dos estímulos usados no estudo para compor as três classes (A, B e C). As formas não representacionais de cada classe eram apresentadas em quantidades variando de um a cinco, em tamanhos grande e pequeno e com disposição espacial alinhada e não alinhada.

\section{Procedimento geral}

A interface do programa utilizado na coleta de dados constitui-se de uma tela com a seguinte configuração. Um retângulo com a palavra "Toque", medindo aproximadamente $2,5 \mathrm{~cm} \times 2,5 \mathrm{~cm}$, localizado no centro da parte superior. Abaixo dele, seis retângulos dispostos lado a lado compõem o que designaremos por "área de construção", em razão de sua função, descrita imediatamente a seguir. Na parte inferior da tela, duas fileiras de quatro retângulos constituem a "área de escolha". Estímulos inicialmente dispostos aleatoriamente na área de escolha, quando tocados com o dedo deslocam-se para a área de construção, posicionando-se da esquerda para a direita. (Ver Figura 2.)

O procedimento compôs-se de quatro fases: uma de pré-teste, uma de treino, uma de testes e uma fase final de seguimento (testes de manutenção).

Nas tentativas de produção de sequências, os estímulos foram apresentados aos pares. Para efeitos de compreensão, a expressão: "pares de estímulos", tal como está sendo usada aqui, refere-se, de fato, a pares de conjuntos de estímulos, estes contendo de um a cinco elementos. Um procedimento de reforço diferencial foi adotado a fim de colocar as respostas sob controle da numerosidade dos estímulos.

Cada tentativa iniciava com a apresentação de um par de (conjuntos) de estímulos dispostos aleatoriamente na área de escolha. O participante era, então, instruído a tocar um estímulo por vez, resposta esta que produzia seu deslocamento para a área de construção. Instruções adicionais como "preste atenção", "fique atento às figuras", "olhe para as duas figuras antes de tocar", foram fornecidas a fim de minimizar a possibilidade de ocorrência de erros. 


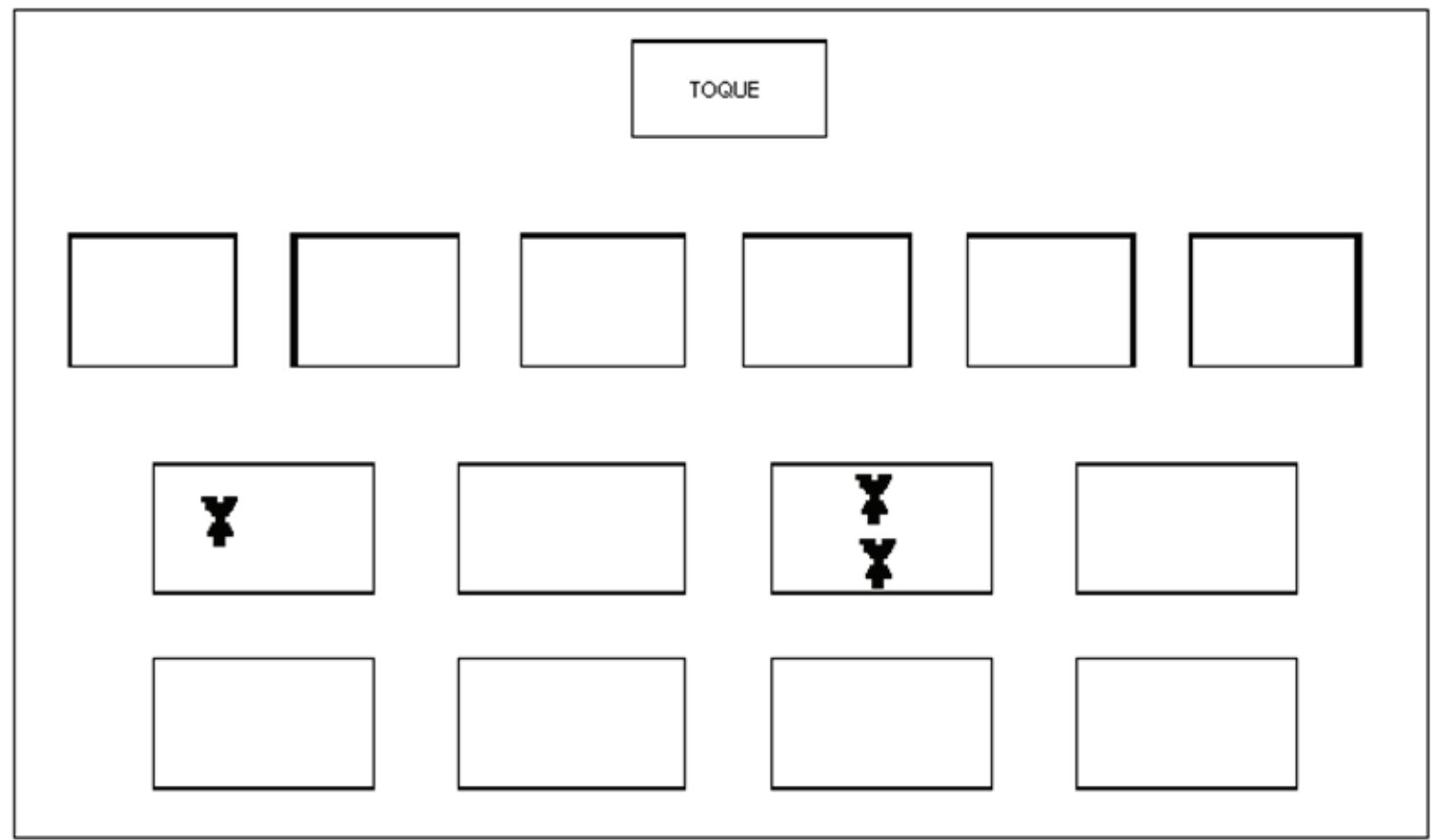

Figura 2. Reprodução de tela do programa usado no experimento. Centralizada na parte superior, encontra-se uma janela com a palavra "toque". Abaixo dela encontram-se as seis janelas da área de construção e, mais abaixo, as oito janelas da área de escolha. Como ilustrado na figura, os estímulos eram apresentados em posições aleatórias na área de escolha. Tocar esses estímulos com o dedo produzia o seu deslocamento para a área de construção.

O número máximo de repetições de uma mesma tentativa previsto para essa fase foi dez. Caso o participante não alcançasse o critério de desempenho (conforme descrição abaixo), ele era re-exposto a até duas vezes o mesmo bloco de tentativas.

Para o treino, adotou-se o procedimento de sobreposição de pares de estímulos. Isto é, os estímulos foram apresentados aos pares. $\mathrm{O}$ treino iniciou-se com a produção da sequência $\mathrm{A} 1 \rightarrow \mathrm{A} 2$; a seguir foi ensinada a sequência $\mathrm{A} 2$ $\rightarrow \mathrm{A} 3$ e assim por diante.

O critério de desempenho adotado previa a seleção dos estímulos em ordem crescente ao longo de três tentativas consecutivas, sem erros. Na fase de treino, respostas corretas produziam uma animação gráfica exibida na tela do computador, mais consequências verbais fornecidas pelo experimentador, como "muito bem", "parabéns", ou "você acertou". Respostas incorretas foram seguidas por um escurecimento da tela durante um período de $3 \mathrm{~s}$, após o qual a tentativa era reapresentada com os estímulos na mesma posição na área de escolha (procedimento de correção).

Sondas foram intercaladas entre as tentativas de treino na razão três para um, isto é, uma sonda a cada três tentativas de treino, desde que nestas não houvesse ocorrência de erro. Nelas, não havia qualquer consequência programada para as respostas. Sua inclusão teve a finalidade de preparar os participantes para a fase de testes, na qual as respostas seriam produzidas em extinção. $\mathrm{O}$ alcance do critério num bloco de tentativas de treino determinava a apresentação do bloco seguinte.

$\mathrm{Na}$ fase seguinte foram conduzidos testes sem consequências diferenciais, de transitividade e conectividade. Detalhes são fornecidos adiante. Caso o participante não alcançasse o critério de desempenho em algum dos testes, a linha de base era retreinada e, a seguir, os testes eram reaplicados.
As sessões foram programadas para durarem 60 minutos, no máximo. Sua duração real foi de vinte minutos em média, o tempo restante tendo sido utilizado para a realização de atividades lúdicas. As sessões ocorriam quatro vezes por semana, sempre no mesmo horário. Ao final de cada sessão, independente de seu desempenho, os participantes receberam brindes. Este procedimento foi adotado como recurso para manter as crianças motivadas a participarem do experimento.

\section{Pré-Teste - Discriminação de quantidades}

Participante e experimentador sentavam-se um frente ao outro a uma mesa sobre a qual eram disponibilizados blocos lógicos (marca FUNBEC). O experimentador separava uma determinada quantidade de peças previamente definida e as empilhava na frente da criança. Após isso, o experimentador perguntava: "quantos têm?", apontando para a pilha de peças, ao que o participante deveria responder oralmente, indicando o número de peças. Considerou-se como critério de participação o acerto igual ou menor a $40 \%$ das tentativas do pré-teste.

\section{Treino de produção de sequências com estímulos adjacentes da classe $A$}

Nesta fase, os participantes foram ensinados a produzir sequências de dois estímulos, todos da classe $\mathrm{A}$, em ordem crescente. $\mathrm{O}$ procedimento adotado foi o de sobreposição de pares de estímulos adjacentes, o qual consiste da apresentação de pares de estímulos em posições aleatórias na área de escolha, reforçando-se a seleção dos estímulos na ordem A1 $\rightarrow$ A2. Alcançado o critério de desempenho com esse par de 
estímulos, nova sequência foi treinada, desta vez A2 $\rightarrow$ A3; depois A3 $\rightarrow$ A4 e, finalmente, A4 $\rightarrow$ A5.

Como descrito anteriormente, após o ensino da produção de sequência com cada par de estímulos foi introduzida uma sonda, isto é, uma tentativa idêntica às de treino, porém, sem consequência programada para resposta correta ou incorreta.

\section{Testes de transitividade}

Estes testes tiveram por objetivos verificar se as relações ensinadas entre os estímulos da classe A apresentariam a propriedade emergente de transitividade e se esta se generalizaria para um novo conjunto de estímulos, os da classe B.

Para o teste, os estímulos da classe A foram apresentados em pares não adjacentes, começando com A1 e A3, depois A1 e A4 e assim sucessivamente, até que todas as combinações possíveis de pares não adjacentes tivessem sido apresentadas. A seleção dos estímulos na ordem crescente seria considerada consistente com o treino e tomada como indicativa de transitividade. Respostas de acordo com esta ordem prevista levavam à apresentação de um novo par de estímulos. Caso contrário, a tentativa era reapresentada, oferecendo-se ao participante nova oportunidade de resposta.

Concluídos os testes de transitividade com os estímulos A, os mesmos testes foram conduzidos com os da classe B e, posteriormente, com os da classe $\mathrm{C}$, com a finalidade de verificar a generalização do repertório aprendido para novos conjuntos de estímulos, os quais não haviam sido apresentados no treino.

\section{Testes de Conectividade}

O teste de conectividade permite verificar a formação de classes de estímulos ordinais derivadas do treino original, pois avalia a substituibilidade entre estímulos de classes diferentes. Estímulos das classes A e B foram apresentados simultaneamente em cada tentativa. Respostas de acordo com esta ordem prevista levavam à apresentação de um novo par de estímulos. Caso contrário, a tentativa era reapresentada, oferecendo-se ao participante nova oportunidade de resposta. Seria considerada consistente com o treino e, portanto, como evidência da propriedade de conectividade da relação entre os estímulos, a produção de sequências tais como $\mathrm{A} 1 \rightarrow \mathrm{B} 2$, $\mathrm{B} 4 \rightarrow \mathrm{A} 5$, etc. Sendo quatro relações do tipo AB, BA, AC, $\mathrm{CA}, \mathrm{BC}$ e $\mathrm{CB}$.

Posteriormente ao teste com os estímulos das classes A e $\mathrm{B}$, também foi testada a conectividade entre os estímulos das classes A e C e, mais tarde, com os das classes B e C. Em seguida, um teste final de conectividade foi aplicado para verificar a conectividade entre três estímulos em sequência das três classes: $\mathrm{A}, \mathrm{B}$ e $\mathrm{C}$, sendo três relações do tipo $\mathrm{ABC}$, BCA e CAB.

\section{Teste de manutenção}

Seis semanas após a conclusão do experimento e sem contato dos participantes com as contingências experimentais, os testes de conectividade foram reaplicados. Aplicou-se um teste de conectividade e, em seguida, um teste de conectividade com três estímulos em sequência, adotando-se o mesmo critério dos testes de conectividade.

\section{Resultados}

A Tabela 2 apresenta os resultados do pré-teste. Todos os participantes apresentaram desempenho igual ou menor que $40 \%$, isto é, de acordo com o critério adotado para sua inclusão no estudo.

Tabela 2. Respostas dos participantes na discriminação de quantidade correspondente no Pré-teste.

\begin{tabular}{cccccc}
\hline \multicolumn{5}{c}{ Participantes } \\
\hline $\begin{array}{c}\text { Número } \\
\text { de itens }\end{array}$ & HEN & WEL & WAL & LIE & DUD \\
1 & $*$ & $*$ & $*$ & - & - \\
2 & $*$ & - & - & - & - \\
3 & - & - & $*$ & $*$ & - \\
4 & - & - & - & - & $*$ \\
5 & - & - & - & $*$ & - \\
& 2 & 1 & 2 & 2 & 1 \\
& $40 \%$ & $20 \%$ & $40 \%$ & $40 \%$ & $20 \%$ \\
\hline
\end{tabular}

Nomes dos participantes: HEN, WEL, WAL, LIE, DUD

$$
\begin{aligned}
*= & \text { Participante respondeu corretamente à quantidade de peças } \\
& \text { naquele item. } \\
-= & \text { Participante respondeu diferente do previsto para aquele item. }
\end{aligned}
$$

Dados do treino são apresentados na Figura 3. Como se vê, todos os cinco participantes alcançaram o critério de desempenho, exigindo diferentes quantidades de treino para a aprendizagem da ordenação dos estímulos da classe A. Os participantes DUD e HEN precisaram de duas exposições a algumas das sondas inseridas entre as tentativas de treino para responderem de acordo com o programado.

A Figura 4 apresenta os resultados dos testes de transitividade com a classe de estímulos "B" e "C". Todos os participantes responderam corretamente na primeira exposição de todos os pares de estímulos dessa classe, exceto os participantes HEN ao par B1B4 e o participante DUD ao par B2B4 na segunda tentativa, na classe de "B" e responderam corretamente na primeira exposição de todos os pares de estímulos da classe "C", exceto os participantes HEN ao par $\mathrm{C} 2 \mathrm{C} 4$ na segunda tentativa.

A Figura 5 apresenta os resultados dos participantes no teste de conectividade com os estímulos da classe $\mathrm{A} / \mathrm{C}$ e $\mathrm{B} / \mathrm{C}$. O participante DUD respondeu prontamente na maior parte dos testes de conectividade e precisou de re-exposição ao par de estímulos C1A4. Todos os participantes também responderam prontamente na primeira tentativa, exceto DUD na relação B1C5 e DUD e HEN na relação C1B2 em segunda tentativa.

Nos testes de conectividade com os estímulos das classes A, B e C, conforme se observa na Figura 6, os participantes responderam prontamente em todas as tentativas, exceto DUD e HEN, que precisaram de re-exposição em três pares 
Número de exposição por participante em cada relação ensinada e nas respectivas sonda

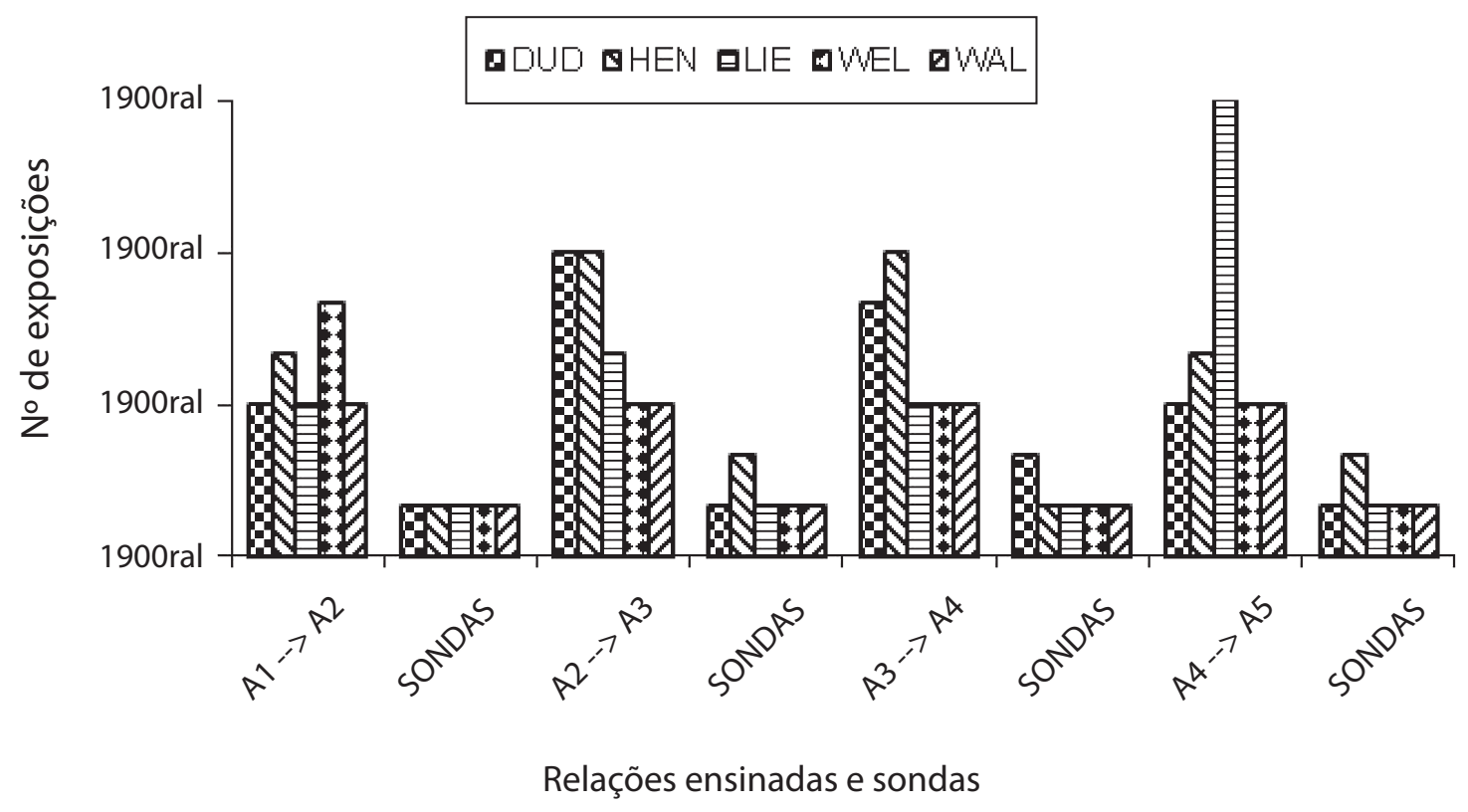

Figura 3. Número total de exposições aos pares de estímulos na fase de treino, por participante.

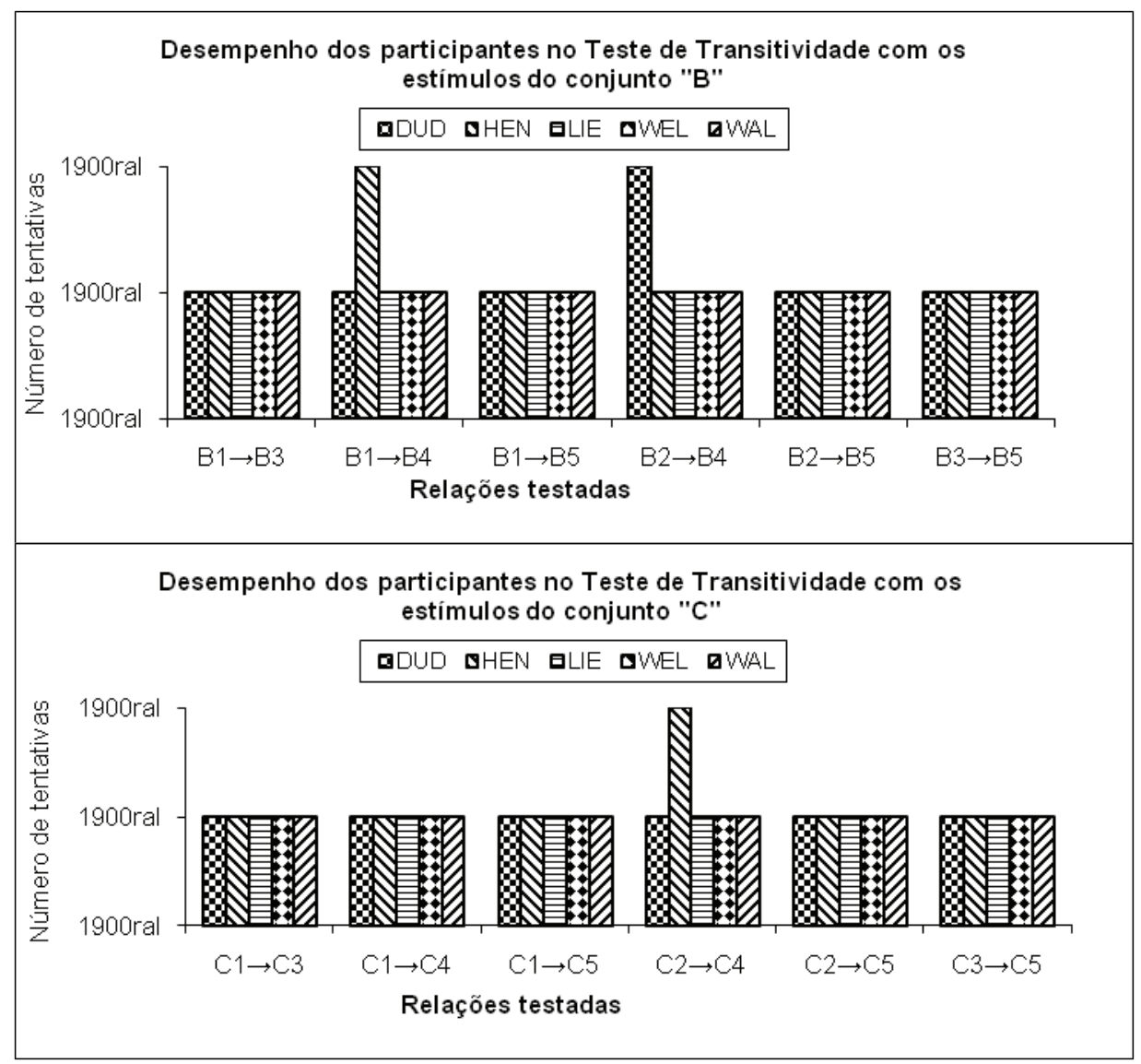

Figura 4. Número total de tentativas aos pares de estímulos nos testes de transitividade do conjunto B e C, por participante. 


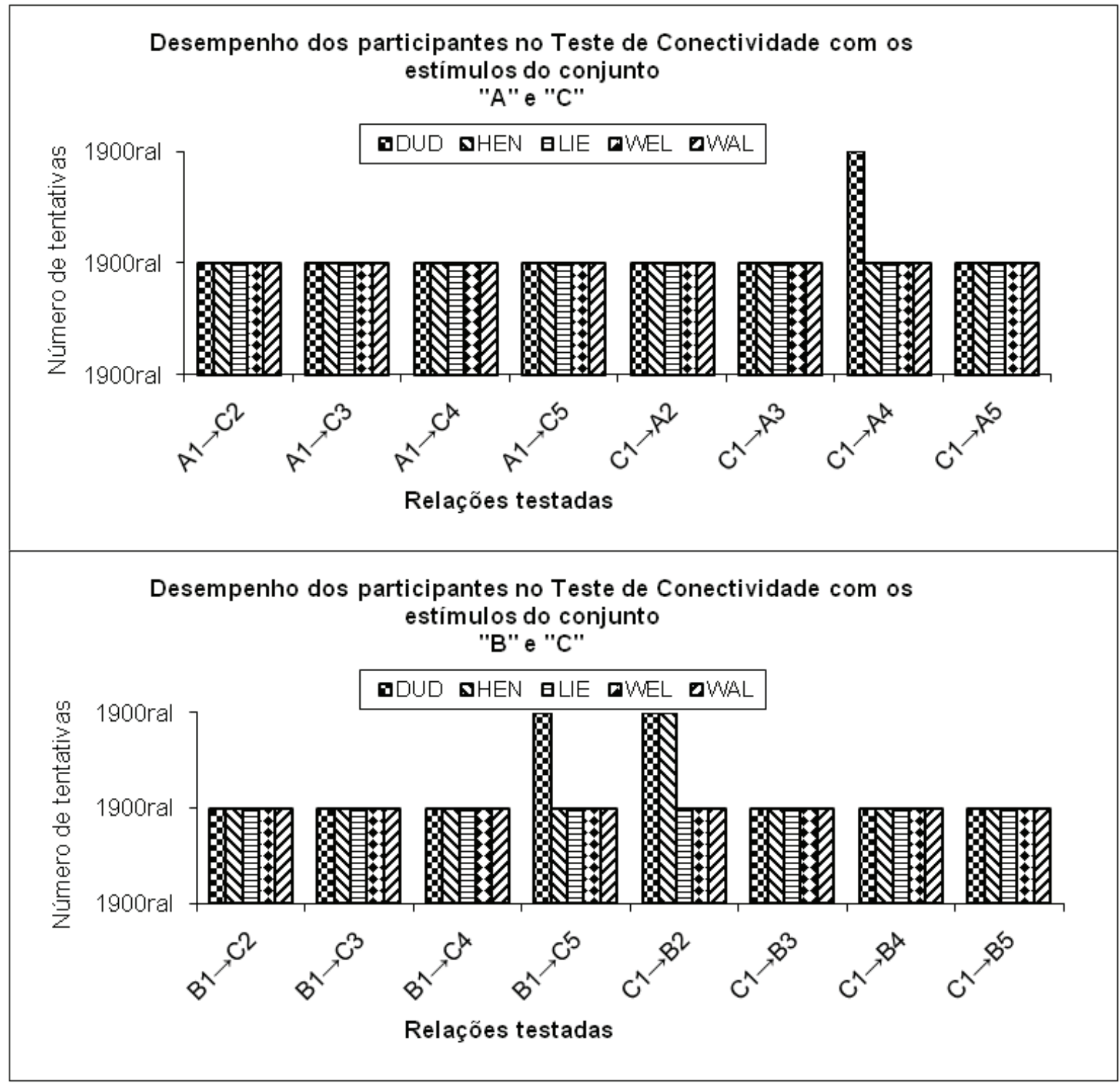

Figura 5. Número total de tentativas aos pares de estímulos nos testes de conectividade das relações A-C e B-C, por participante.

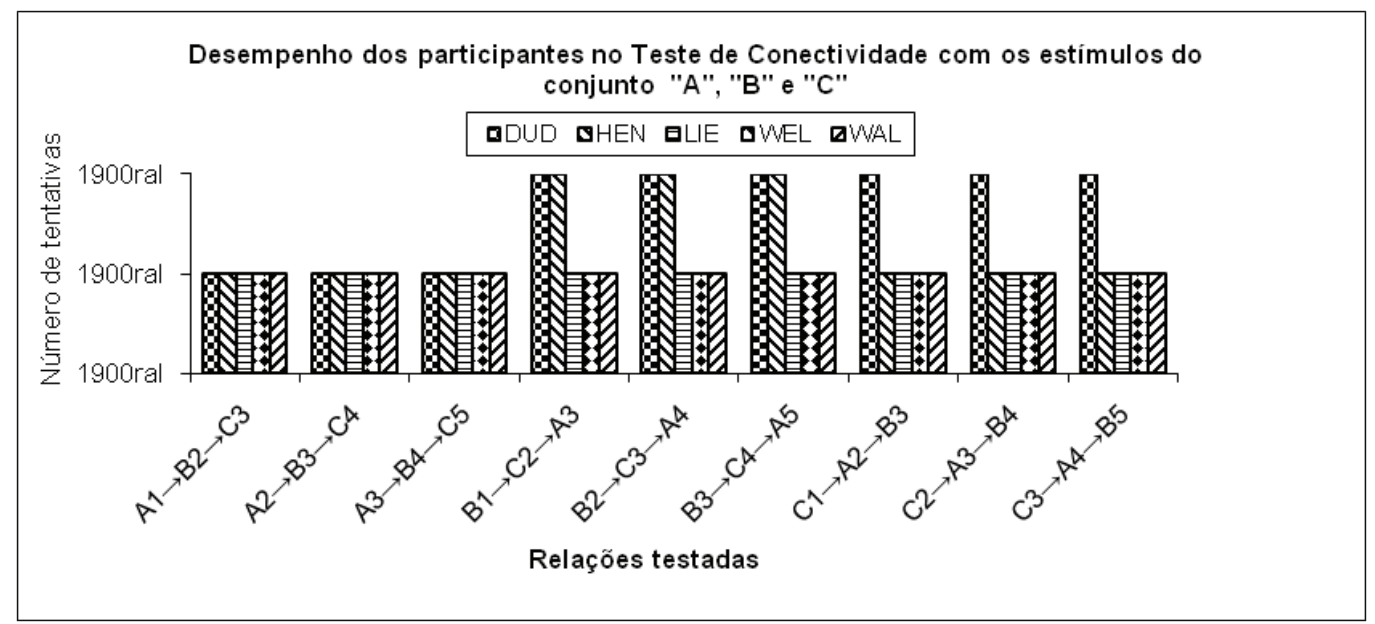

Figura 6. Número total de tentativas nos testes de conectividade com os estímulos dos três conjuntos: "A", B" e "C". 
de estímulos, respectivamente. DUD ainda respondeu na segunda tentativa a três outros pares de estímulos.

A Figura 7 apresenta os resultados nos testes de conectividade aplicados na fase "Testes de manutenção" com dois estímulos em sequência. Nela não constam os dados de DUD, pois ela abandonou o estudo. Na Figura 7 observa-se que os participantes WEL e WAL responderam prontamente a todos os pares de estímulos. HEN, com exceção de um par (A1B3), respondeu prontamente em todos os outros.

Todos os participantes responderam prontamente às relações AC/CA. Porém, a participante LIE não respondeu a quatro pares (A1C2, A1C5, C1A2 e C1A5 - ver Figura 7) da relação $\mathrm{AC} / \mathrm{CA}$.

Todos os participantes responderam prontamente às relações BC/CB, porém, a participante LIE não respondeu aos pares de estímulos na relação $\mathrm{BC}$ e respondeu prontamente aos pares C1B3 e C1B5 e não respondeu aos demais pares dessa mesma relação.

\section{Discussão}

Este estudo mostrou a possibilidade de se produzirem sequências não diretamente ensinadas a partir do ensino de uma única sequência, diferentemente do observado nos estudos dessa área que têm obtido comportamento ordinal emergente a partir do ensino de, no mínimo, duas sequências (e.g. Souza e cols., 2005; Stromer \& Mackay, 1993; Stromer e cols., 1993; Verdu; Souza; Lopes Junior, 2006). Crianças de cinco anos que não apresentavam respostas bem estabelecidas de tacto (conforme Skinner, 1992) para numerosidades de 1 a 5 e para quem tais respostas (ou qualquer habilidade relacionada à contagem) não foram explicitamente ensinadas, aprenderam a ordenar conjuntos com aqueles valores e generalizaram esse desempenho para conjuntos de estímulos não treinados. Além disso, exibiram desempenhos que permitem inferir a emergência de propriedades definidoras de classes ordinais, de acordo com a definição proposta por Green e colaboradores (1993), replicando dados de pesquisas anteriores (Souza e cols., 2005; Holcomb e cols., 1997; Stromer e Mackay, 1993 e Stromer e cols., 1993).

A possibilidade que a aprendizagem do repertório de ordenação e a formação de classes ordinais tenham derivado de um processo de generalização simples a partir do treino da primeira sequência pode ser levantada. No entanto, a formação de classes ordinais implica um repertório derivado que não guarda similitude com o tipo de contingência do treino da primeira sequência. Já o repertório de ordenação para ser generalizado necessitaria da existência de propriedades comuns que controlassem o responder dos participantes diante dos diferentes conjuntos de estímulos usados para compor as diferentes sequências. Essa possibilitada é analisada na discussão que se segue.

Devido à natureza da tarefa proposta às crianças desse estudo, duas propriedades de estímulo foram, necessariamente, sobrepostas: posição e numerosidade. Portanto, não há como sabermos exatamente em que medida cada uma delas ou a interação entre ambas controlou as respostas. Portanto, conduziremos a discussão em duas linhas distintas, cada uma delas considerando separadamente possíveis efeitos de uma ou outra daquelas propriedades, até que novas pesquisas tornem mais claros os efeitos de cada uma, bem como os de sua interação.

\section{Considerando a numerosidade}

O controle experimental sobre as dimensões tamanho e disposição espacial dos elementos nos conjuntos faz da numerosidade uma possível dimensão sob cujo controle os participantes responderam, além da posição. Considerando que, como demonstrado nos dados do pré-teste, os participantes não contavam, discutiremos a relação entre a numerosidade e a contagem. Embora Green (1993) tenha observado a formação de classes de equivalência compostas por números falados, escritos e conjuntos em jovens com déficit de linguagem que não contavam, pesquisas com crianças em idade pré-escolar e escolar apresentam evidências que vão desde a simples correlação entre a contagem e a equiparação de conjuntos (Mix, 1999; Prado, 2008), entre a contagem e o ulterior desempenho escolar em matemática (Aunola, Leskinen, Lerkkanen, \& Nurmi, 2004), até as que apontam a contagem como pré-requisito para a aprendizagem dessas e outras habilidades (Spradlin, Cotter, Stevens \& Friedman, 1974; Wang, Reskink \& Boozer, 1971).

Se o desempenho dos participantes nos testes de transitividade e conectividade não pode ser atribuído à contagem, como interpretá-lo? Como tem sido demonstrado por diversas pesquisas, a discriminação de numerosidades de 1 a 4 prescinde da contagem (e.g. Antell \& Keating, 1983; Hauser \& Carey, 2003; Hauser \& Spelke, 2004; Trick \& Pylyshyn, 1994), o que não ocorre com numerosidades maiores (e.g. Hauser \& Spelke, 2004). A introdução de conjuntos com cinco ou mais elementos, portanto, permitiria verificar se a contagem é ou não necessária para formação de classes ordinais com esses valores.

\section{Considerações sobre a posição dos estímulos}

O procedimento de ensino utilizado mostrou-se eficiente para produzir sequências não diretamente ensinadas e a emergência de classes ordinais, corroborando os dados de Souza e cols. (2005), Holcomb e cols. (1997), Stromer e Mackay (1993) e Stromer e cols. (1993). Todos os participantes responderam prontamente à maioria das tentativas nos testes de transitividade e conectividade. Os participantes responderam consistentemente nos testes de conectividade, indicando que os estímulos tornaram-se funcionalmente equivalentes (substituíveis), ou seja, estímulos que ocupavam uma determinada posição em uma sequência quando intercalados com estímulos de outras sequências exerciam a mesma função estabelecida segundo a ordem envolvida no treino.

Como afirmaram Ribeiro e cols. (2005):

Classes ordinais são formadas por estímulos provenientes de sequências independentes e que ocuparam a mesma posição ordinal. Assim, para avaliação do potencial transitivo das relações ordinais, o exame da substituição de estímulos em provas mistas, em que estímulos provenientes de sequências 


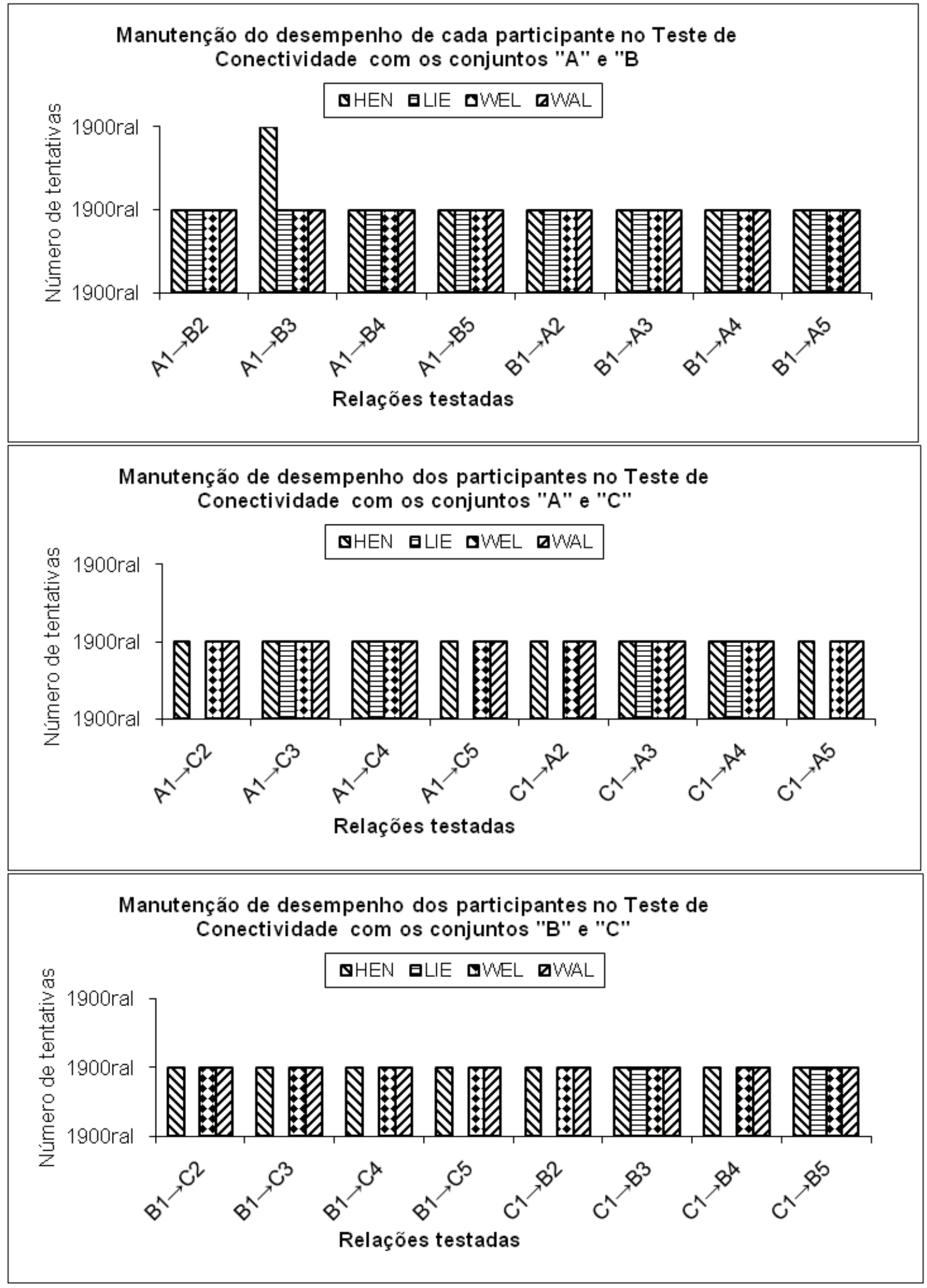

Figura 7. Número total de tentativas nos testes de conectividade aplicados na fase de manutenção das relações A-B, A-C e B-C. 
diferentes devem ser ordenados, tornou-se a estratégia de exame mais comum. Além disto, as provas mistas permitem o exame da propriedade conectividade, que implica que todos os pares de itens dentro de uma sequência estão relacionados entre si. A propriedade conectividade é inferida se todos os pares possiveis, adjacentes e não adjacentes, forem ordenados adequadamente (p. 129).

No que diz respeito à substituibilidade de estímulos de diferentes sequências, este tem sido para muitos autores (Assis \& Costa, 2004; Galy, Camps \& Melan, 2003; Green e cols., 1993; Mackay, Kotlarchyk \& Stromer, 1997; Sampaio \& Assis, 2005; Stromer \& Mackay, 1993) um parâmetro para se avaliar propriedades ordinais entre estímulos.

Além disso, a extensão para sequências mais longas com três estímulos produziu efeitos semelhantes, evidenciando um fortalecimento do controle de estímulos em sequências. Isso amplia os dados da literatura na área.

Nos testes de manutenção, três participantes (HEN, WEL e WAL) responderam prontamente a maior parte das tentativas. Este desempenho demonstrou que para estes três participantes o repertório se manteve após um período de tempo sem contato com as contingências experimentais, replicando os resultados de Souza e cols. (2005).

A participante LIE não respondeu a maior parte dos testes de manutenção, e foi exposta aos testes de transitividade, supostamente mais simples por se tratar de relações de estímulos intraclasse. Em seguida, a mesma participante voltou a ser exposta aos testes de conectividade. Apesar da re-exposição aos testes de manutenção, após retorno ao teste de transitividade com os estímulos dos três conjuntos ("A", "B" e "C"), a participante LIE respondeu prontamente somente no teste de conectividade AB e BA. Nos outros testes de conectividade o desempenho permaneceu irregular.

Analisando-se as respostas nos testes de manutenção da participante LIE, que não estavam de acordo com a programação ensinada, não há evidências de controle por propriedades específicas dos estímulos. Deste modo, o desempenho de LIE pode ter sido afetado por algumas das contingências de sua historia pré-experimental.

Os resultados do estudo indicam que o treino de uma sequência de estímulos, com a numerosidade como propriedade discriminativa relevante, pode levar à emergência de novas sequências sem qualquer treino adicional. Além da óbvia economia de tempo que a necessidade de treinar apenas uma sequência de estímulos pode permitir ao processo de aquisição de comportamento ordinal emergente, este procedimento ainda se mostra relevante também para testar o grau de generalização deste tipo de comportamento a partir de diferentes tipos de treinos (por exemplo: encadeamento versus sobreposição).

$\mathrm{O}$ efeito do treino de uma sequência com estímulos com a numerosidade como propriedade discriminativa relevante, sugerido no estudo de Souza e cols. (2005) e observado no presente estudo, precisa ser analisado em um estudo que compare a aquisição de comportamento ordinal emergente após treinos com estímulos com essa propriedade e com estímulos sem ela. A sobreposição dessa propriedade (numerosidade) à contingência de discriminação simples baseada no responder sequencial, torna ainda mais clara a possibilidade de se estu- dar a aquisição de comportamentos conceituais numéricos no contexto dos estudos sobre comportamento ordinal emergente conforme a proposta de Green e cols. (1993).

No momento, grande parte da literatura tem investigado a aquisição de relações numéricas a partir de discriminações condicionais no contexto de emparelhamento com o modelo (Carmo, 2002; Kahhale, 1993; Prado \& de Rose, 1999). Com resultados que mostram a aquisição de relações numéricas com a utilização de procedimentos a partir de discriminações simples, mostra-se que processos envolvidos em alguns comportamentos matemáticos como a contagem e ordenação, podem ser analisados por meio de arranjos de contingências simples. O aprofundamento na compreensão de como contingências simples de responder sequencial, sobrepostas à propriedade de numerosidade dos estímulos, proporcionam a emergência de comportamento ordinal, pode permitir o desenvolvimento de um procedimento eficaz para uma tecnologia de ensino de alguns daqueles repertórios.

Pretende-se em um próximo estudo replicar esse experimento com crianças com dificuldades de aprendizagem matemática. A replicação dos resultados com esses alunos poderá indicar com mais segurança a eficiência do procedimento de ensino. Testes de generalização com estímulos do ambiente natural do participante podem ser aplicados para avaliar a generalidade dos dados. Uma entrevista pós-experimental poderá auxiliar a identificar quais propriedades dos estímulos controlaram a resposta dos participantes.

Respostas a outras questões também poderão ser buscadas por pesquisas futuras. O procedimento adotado neste estudo poderia fazer com que as crianças tratassem como numericamente equivalentes conjuntos que ocuparam inicialmente a mesma posição ordinal? E ainda, um treino adicional de produção de sequências com numerais faria com que estes fossem incluídos nas mesmas classes de equivalência dos conjuntos? Em caso afirmativo, estaríamos replicando, com crianças, dados produzidos por Maydak, Stromer, Mackay e Stoddard (1995), em pesquisa com adultos com atraso de desenvolvimento cognitivo.

O procedimento mostrou-se eficaz no ensino de uma importante habilidade que pode estar na base do aprendizado de outras habilidades matemáticas mais complexas. Mas pesquisas ulteriores também são necessárias para que se possa determinar com maior precisão a extensão em que a ordenação de conjuntos pode beneficiar crianças pré-escolares em suas aprendizagens escolares futuras.

\section{Referências}

Antell, S. E., \& Keating, D. P. (1983). Perception of numerical invariance in neonates. Child Development, 54, 695-701.

Assis, G. J. A., \& Costa, L. C. A. (2004). Emergência de relações ordinais em crianças. Interação em Psicologia, 8, 199-216.

Aunola, K., Leskinen, E., Lerkkanen, M. K., \& Nurmi, J. E. (2004). Developmental dynamics of math performance from preschool to grade 2. Journal of Educational Psychology, 96, 699-713.

Carmo, J. (2002). Definições operacionais de habilidades matemáticas elementares. Em H. Guilhardi., M. B. B. Madi., P. P. Queiroz., \& M. C. Scoz (Orgs.), Sobre comportamento e cognição (pp.181-191), Santo André: ESETec. 
Galy, E., Camps, J. F., \& Melan, C. (2003). Sequence class formation following learning of short sequences. The Psychological Record, 53, 635-645.

Green, G. (1993). Stimulus Control Technology for Teaching Number-Quantity Equivalences. Proceedings of the 1992 Conference of the National Association for Autism (Australia), 51-63, Melbourne, Australia: Victoria Autistic Children's and Adults Association, Inc.

Green, G., Stromer, R., \& Mackay, H. (1993). Relational learning in stimulus sequences. The Psychological Record, 43, 599-616.

Hauser, M. D., \& Carey, S. (2003). Spontaneous representations of small number of objects by rhesus macaques: Examinations of content and format. Cognitive Psychology, 47, 367-401.

Hauser, M. D., \& Spelke, E. (2004). Evolutionary and developmental foundations of human knowledge: A case study of mathematics. Em: M. S. Gazzaniga (Org.), The Cognitive Neurosciences (pp. 853-864). Cambridge: MIT Press.

Holcomb, W. L., Stromer, R., \& Mackay, H. A. (1997). Transitivity and emergent sequence performances in young children. Journal of Experimental Child Psychology, 65, 96-124.

Kahhale, E. M. S. P. (1993). Comportamento matemático: formação e ampliação do conceito de quantidade e relações de equivalência. Tese de Doutorado, Instituto de Psicologia, Universidade de São Paulo, São Paulo.

Mackay, H. A., Kotlarchyk, B. J., \& Stromer, R. (1997). Stimulus classes, stimulus sequences, and generative behavior. Em D. M. Baer., \& E. M. Pinkston (Eds.), Environment and Behavior (pp. 124-137). Boulder, CO: WestviewPress.

Maydak, M., Stromer, R., Mackay, H. A., \& Stoddard, L. (1995). Stimulus classes in matching to sample and sequence production: The emergence of numeric relations. Research in Developmental Disabilities, 16, 179-204.

Mix, K. S. (1999). Similarity and numerical equivalence: Appearances count. Cognitive Development, 14, 269-297.

Prado, P. S. T. (2008). Análise correlacional entre contagem e equiparação de conjuntos. Em: W. C. M. P. SILVA (Org.). Sobre comportamento e cognição: reflexões epistemológicas e conceituais, considerações metodológicas, relatos de pesquisa (pp. 185-200). Santo André: ESETec.

Prado, P. S. T., \& De Rose, J. C. C. (1999). Conceito de número: uma contribuição da análise comportamental da cognição. Psicologia: Teoria e Pesquisa, 15, 227-235.

Ribeiro, M. P. L., Assis G. J. A., \& Enumo, S. R. F. (2005). Controle do comportamento por relações ordinais: questões conceituais e metodológicas. Em E. B. Borloti., S. R. F. Enumo., \& M. L. P. Ribeiro (Orgs.), Análise do comportamento: teorias e práticas (pp. 117-132). Santo André: ESETec.
Santos, A. S L., Silva, A. M. M. V., Baptista, M. Q. G., \& Assis, G. J. A. (1997). REL 1.0: Sistema computadorizado para o ensino de discriminações simples e condicionais. Resumos de Comunicações Científicas, XXVII Reunião Anual de Psicologia, Sociedade Brasileira de Psicologia, Ribeirão Preto-SP.

Sampaio, M. E. C., \& Assis, G. J. A. (2005). Equivalência de estímulos sequenciais em portadores de necessidades educacionais especiais. Acta Comportamentalia, 13, 111-143.

Sidman. M. (1994). Equivalence relations and behavior: a research story. Boston: Authors Cooperative.

Skinner, B.F. (1992). Verbal Behavior. Acton, Massachusetts: Copley (originalmente publicado em 1957).

Souza, R. D. C., Assis, G. J. A., \& Magalhães, P. G. S. (2005). Equivalência numérica em crianças surdas. Temas em Psicologia, 13, 113-127.

Spradlin, J. E., Cotter, V. W., Stevens, C., \& Friedman, M. (1974). Performance of mentally retarded children on pre-arithmetic tasks. American Journal of Mental Deficiency, 78(4), 397-403.

Stromer, R., \& Mackay, H. A. (1992). Some effects of presenting novel stimuli on a child's sequence production. Experimental Analysis of Human Behavior Bulletin, 10, 21-25.

Stromer, R., \& Mackay, H. A. (1993). Human sequential behavior: relations among stimuli, class formation, and derived sequences. The Psychological Record, 43, 107-131.

Stromer, R., Mackay, H. A., Cohen, M., \& Stoddard, L. T. (1993). Sequence learning in individuals with behavioural limitations. Journal of Intellectual Disability Research, 37, 243-261.

Trick, L., \& Pylyshyn, Z. (1994). Why are small and large numbers enumerated differently? A limited capacity preattentive stage in vision. Psychological Review, 101, 80-102.

Verdu, A. C. M. A., Souza, D. G., \& Lopes Jr. J. (2006). Formação de classes ordinais após a aprendizagem de sequências independentes. Estudo de Psicologia, 11, 87-99.

Wang, M. C., Resnick, L. B., \& Boozer, R. F. (1971). The sequence of development of some early mathematical behaviors. Child Development, 42, 1767-1778. 